\title{
Perfil socioeconômico e demográfico municipal em relação à mortalidade hospitalar de idosos por sexo
}

\author{
Camila Oliveira*, Giovane Viana Campos**, Saulo de Lima Pereira***, Gisele Viana Campos****, \\ Ana Cristina Viana Campos*****
}

*Enfermeira, Especialista em Enfermagem em Enfermagem Urgência, Emergência e Trauma pelo Instituto de Educação Continuada Pontifícia Universidade Católica de Minas Gerais (IEC PUC Minas), **Turismólogo, Aperfeiçoamento em análise de dados demográficos (Censo IBGE 2010), ${ }^{* * *}$ Enfermeiro do Hospital Vaz Monteiro, Lavras, Minas Gerais, ****TerapeutaOcupacional na Estratégia de Saúde da Família de Lavras, Minas Gerais, ${ }^{* * * *}{ }^{*}$ Cirurgiä-dentista, Doutoranda em Saúde Coletiva pela Faculdade de Odontologia da Universidade Federal de Minas Gerais (UFMG)

\section{Resumo}

O objetivo deste estudo foi descrever o perfil socioeconômico e demográfico dos municípios mineiros em relação à mortalidade hospitalar de idosos por sexo. Estudo ecológico com dados secundários do Departamento de Informática do Sistema Único de Saúde e do Atlas Eletrônico de Desenvolvimento Humano com os 853 municípios de Minas Gerais. Foi utilizada a análise de segmentação para agrupar os municípios por meio dos indicadores socioeconômicos e demográficos. O agrupamento 1 reuniu municípios com piores indicadores socioeconômicos, maior densidade demográfica e maior índice de Gini. O agrupamento 2 reuniu os outros municípios, com maiores médias, portanto classificados como de melhores indicadores. Houve apenas diferenças relativas entre os idosos, homens e mulheres, no conjunto formado pelos dois agrupamentos. Apenas com esses dados, conclui-se que os municípios mineiros não são diferentes em relação aos indicadores municipais e mortalidade hospitalar de ambos os sexos.

Palavras-chave: mortalidade hospitalar, idoso, indicadores sociais.

\section{Abstract \\ Socioeconomic and demographic profile of a district in relation to hospital mortality of elderly by sex}

Our aim was to describe the socioeconomic and demographic profile of districts of Minas Gerais regarding the mortality rate of the elderly by sex. Ecological study used secondary data from the Department of Informatics at the Unified Health System and the Electronic Atlas of Human Development in the 853 cities of Minas Gerais. The segmentation analysis was used to group the cities through socioeconomic and demographic indicators. The grouping 1 was composed of cities with the worst socioeconomic indicators, higher population density and higher Gini index. Grouping 2 composed of 
other cities with higher averages, therefore classified as better indicators. There were only relative differences between elderly men and women in the set composed of the two groupings. Our data indicated that the cities of Minas Gerais are not different regarding municipal indicators and mortality of both sexes.

Key-words: hospital mortality, elderly, social indicators.

\section{Resumen}

\section{Perfil socioeconómico y demográfico municipal con respecto a la mortalidad hospitalaria de personas de edad por sexo}

El objetivo de este estudio fue describir el perfil socioeconómico y demográfico de los distritos de Minas Gerais con respecto a la tasa de mortalidad de personas de edad por sexo. Estudio ecológico utilizó datos secundarios del Departamento de Informática del Sistema Único de Salud y el Atlas Electrónico de Desarrollo Humano de las 853 ciudades de Minas Gerais. El análisis de segmentación se utilizó para agrupar las ciudades a través de los indicadores socioeconómicos y demográficos. El agrupamiento 1 reunió ciudades con peores indicadores socioeconómicos, mayor densidad de población y el aumento del índice de Gini. El agrupamiento 2 ha sido formado por otras ciudades con promedios más altos, por lo tanto clasificados como mejores indicadores. Encontramos apenas diferencias relativas entre los hombres y las mujeres, en el conjunto formado por los dos agrupamientos. Sólo con estos datos, se concluye que los distritos de Minas Gerais no son diferentes con respecto a los indicadores municipales y la mortalidad de ambos sexos.

Palabras-clave: mortalidad hospitalaria, ancianos, indicadores sociales.

\section{Introdução}

O envelhecimento populacional é, hoje, um proeminente fenômeno mundial marcado pelo crescimento mais elevado da populaçáo idosa com relação aos demais grupos etários. Além disso, a proporção de indivíduos com 80 anos ou mais de idade, também está aumentando, alterando a composição etária dentro do próprio grupo. Isso leva a uma heterogeneidade desse segmento populacional [1].

Apesar das tendências de redução das taxas de mortalidade infantil e aumento da esperança de vida ao nascer, dados nacionais de 2005 mostraram que existem diferenças deste último indicador entre os sexos, sendo de 68,7 anos para os homens e 76,2 anos para as mulheres [2]. Entre as causas de morte, as doenças do aparelho circulatório aparecem como principal grupo em ambos os sexos, seguida pelas doenças do aparelho respiratório e neoplasias [3].

Outro aspecto da questão do envelhecimento é a feminização da velhice e suas implicaçóes em termos de políticas públicas, pois uma grande parte das mulheres é viúva, vive só, sem experiência de trabalho no mercado formal e é menos educada [4].

No Brasil, a preocupação com os aspectos demográficos do envelhecimento de sua população é relativamente recente. A questão foi primeiramente levantada nos estudos sobre as consequências demográficas da queda da natalidade [5]. A análise das diferenças sociais da mortalidade na metrópole de São Paulo deve considerar as consequências estruturais das opçôes de desenvolvimento que priorizam o econômico em detrimento do social e favorecem a contínua concentraçáo de renda e o impacto da miséria e da exclusão social na saúde das populações adultas do município. Um estudo em São Paulo mostrou que diferenças sociais da mortalidade devem levar em consideração as estruturas sociais que ainda priorizam os aspectos econômicos e favorecem a contínua concentração de renda, bem como o impacto da miséria e da exclusão social na saúde das populaçôes adultas do município [6].

O novo padrão demográfico brasileiro é marcado por diferenças geográficas, socioeconômicas e progressivos declínios das taxas de fecundidade e mortalidade, alteração da estrutura etária e aumento da proporção de idosos. Nesse sentido, o objetivo do presente estudo foi descrever o perfil socioeconômico e demográfico dos municípios mineiros em relação à mortalidade hospitalar de idosos por sexo.

\section{Material e métodos}

Trata-se de um estudo ecológico com utilização de dados secundários obtidos no Departamento de Informática do Sistema Único de Saúde (Datasus) e do Atlas Eletrônico de Desenvolvimento Humano, sobre indicadores socioeconômicos dos municípios 
do Estado de Minas Gerais para o ano de que contém os dados referentes ao censo brasileiro do ano de 2000.

O indicador considerado a variável dependente para o presente estudo foi a taxa de mortalidade de idosos (indivíduo com idade igual ou superior a 60 anos) do ano de 2008. As variáveis independentes foram: taxa de alfabetização, esperança de vida ao nascer, densidade demográfica, Índice de Desenvolvimento Humano (IDH) e os índices de Gini, de Theil e Mineiro de Responsabilidade Social (IMRS); e de saúde: acesso a internação hospitalar, média de consultas médicas, cobertura da Estratégia de Saúde da Família (ESF) [7-9].

O IMRS, elaborado pela Fundação João Pinheiro, é um indicador que expressa o nível de desenvolvimento de cada município mineiro. Foi desenvolvido para avaliar a situação desses municípios, contemplando nove dimensôes, a saber: 1) renda, 2) saúde, 3) educação, 4) segurança pública, 5) gestáo, 6) habitação e meio ambiente, 7) cultura e desporto, 8) lazer. Na construção do IMRS os indicadores de cada tema são transformados em índices, com valores entre 0 e 1 , obtido através da média ponderada dos índices dos indicadores que o compóem [10].

Inicialmente realizou-se a análise descritiva de todas as variáveis em questão. A análise de segmentação denominada agrupamento de dados pelo método K-médias foi realizada para segmentação dos municípios a partir dos indicadores socioeconômicos, demográficos e de saúde. A análise de agrupamentos pressupóe a formação de um conjunto de objetos no qual cada elemento está mais próximo e é mais similar a elementos dentro do agrupamento do que qualquer outro fora do grupo. Dado um número de clusters previamente determinado, calcula-se os pontos que representam os centroides ("centros") estes clusters e que são espalhados homogeneamente e movidos, heuristicamente, até alcançar um equilíbrio estático [11].

O banco de dados foi construído no programa Statistical Package for the Social Sciences for Windows (SPSS) versão 17. Foi realizada uma comparação simples entre os agrupamentos e as taxas de mortalidade do sexo masculino e do sexo feminino.

\section{Resultados}

Em média, os municípios mineiros apresentam aproximadamente 60 habitantes por $\mathrm{km}^{2}$, alto IDH $(0,72 \pm 0,06)$, alta esperança de vida ao nascer de $(69,52 \pm 3,39)$, alta taxa de alfabetização $(81,78 \pm 8,18)$, grande desigualdade social (Índice de Gini $=0,55 \pm 0,05$ e Índice de Theil $=0,51 \pm 0,10)$, mas um bom desenvolvimento regional (IMRS $=0,59 \pm 0,08)$. A cobertura da Saúde da Família e a taxa de internação hospitalar foram os indicadores que apresentaram maior variação entre os municípios mineiros. A cobertura média foi de $83,41 \%( \pm 28,88)$, sendo que em alguns municípios não há cobertura da ESF $(0,0 \%)$ e em outros a cobertura está sobrestimada $(185,44 \%)$. Em relação à internação, os valores variam entre mínimo de 0,51 e máximo de 212,48 .

A análise de segmentação resultou em dois agrupamentos. A Tabela I mostra a média e desvio-padrão de todos os indicadores para ambos os agrupamentos, sendo que os indicadores IDH, Índice de Gini, Índice de Theil são muito semelhantes entre os grupos.

Tabela I - Média e desvio-padrão dos indicadores socioeconômicos e demográficos entre os agrupamentos ( $N=853$ ). Minas Gerais, 2009.

\begin{tabular}{lcc}
\hline \multicolumn{1}{c}{ Indicadores } & Agrupamento 1 & Agrupamento 2 \\
\hline Esperança de vida & $67,77( \pm 3,08)$ & $71,85( \pm 2,13)$ \\
Taxa de alfabeti- & $77,30( \pm 7,83)$ & $87,79( \pm 3,38)$ \\
zação & & \\
Densidade demo- & $63,91( \pm 8,59)$ & $53,90( \pm 4,61)$ \\
gráfica & $0,72( \pm 0,06)$ & $0,72( \pm 0,06)$ \\
IDH & $0,56( \pm 0,05)$ & $0,55( \pm 0,05)$ \\
Índice de Gini & $0,51( \pm 0,09)$ & $0,51( \pm 0,10)$ \\
Índice de Theil & $0,55( \pm 0,07)$ & $0,64( \pm 0,05)$ \\
IMRS & $44,78( \pm 33,98)$ & $21,47( \pm 19,49)$ \\
Internação hospi- & \\
talar & $92,21( \pm 23,61)$ & $71,57( \pm 31,04)$ \\
Cob. ESF & $1,98( \pm 1,21)$ & $2,15( \pm 1,15)$ \\
Méd. cons. mé- & \\
dicas &
\end{tabular}

IDH: Índice de Desenvolvimento Humano; IMRS: Índice Mineiro de Responsabilidade Social; Méd. Cons. Médicas: Média de consultas médicas; Cob.ESF: Cobertura da Estratégia de Saúde da Família. Fonte dos dados: Departamento de Informática do Sistema Único de Saúde - DATASUS e Atlas Eletrônico do Desenvolvimento Humano.

A Tabela II mostra que há diferenças relativas apenas entre os idosos, homens e mulheres, no conjunto formado pelos dois agrupamentos. 
Tabela II - Comparação entre os agrupamentos em relação à taxa de mortalidade de idosos por sexo $(\mathrm{N}=$ 853). Minas Gerais, 2009.

\begin{tabular}{cllll}
\hline \multirow{2}{*}{ Sexo } & \multirow{2}{*}{$\begin{array}{c}\text { Agrupa- } \\
\text { mentos }\end{array}$} & $N(\%)$ & \multicolumn{2}{c}{$\begin{array}{c}\text { Mortalidade de } \\
\text { idosos }\end{array}$} \\
\cline { 4 - 5 } & & & Média & IC 95\% \\
\hline \multirow{2}{*}{ Masculino } & 01 & $489(57,32)$ & 6,26 & {$[6,08-6,45]$} \\
Feminino & 02 & $364(42,68)$ & 6,25 & {$[6,04-6,46]$} \\
& 01 & $489(57,32)$ & 4,92 & {$[4,74-5,10]$} \\
& 02 & $364(42,68)$ & 4,71 & {$[4,56-4,86]$} \\
\hline
\end{tabular}

Fonte dos dados: Departamento de Informática do Sistema Único de Saúde - DATASUS e Atlas Eletrônico do Desenvolvimento Humano.

\section{Discussão}

Este trabalho buscou contribuir com o entendimento do envelhecimento e saúde do idoso e suas peculiaridades medidas pelos indicadores municipais de Minas Gerais. Entende-se que a existência de importantes desafios colocados por esse processo à sociedade devem ser referenciados a uma realidade social complexa.

A literatura destaca o impacto do crescimento da população idosa sobre a razão de dependência demográfica, sobre os gastos de previdência, gastos de saúde, condiçôes de saúde e mortalidade da população idosa [1-2,12].

De modo geral, os municípios mineiros apresentam indicadores sociais comparáveis como taxa de alfabetização $(81,78 \% \pm 8,18)$, IMRS $(0,59 \pm$ $0,08)$. O IDH mineiro é pouco superior $(0,72 \pm$ $0,06)$ ao brasileiro $(0,71)$, e é usado como referência da qualidade de vida e desenvolvimento sem se prender apenas em índices econômicos $[13,14]$. Juntos, esses indicadores se relacionam entre si e são utilizados para demonstrar o grau de desenvolvimento nos aspectos sociais e econômicos.

Dos 15 países do mundo nos quais a distância entre ricos e pobres é maior, 10 estão na América Latina e Caribe. O Brasil tem o terceiro pior Índice de Gini $(0,56)$ [15], e os municípios mineiros seguem a média nacional $(0,55)$.

Em relação aos indicadores de saúde, a cobertura da Estratégia de Saúde da Família apresenta média igual a $83,41 \%( \pm 28,88)$, baixa porcentagem média de internaçáo hospitalar 34,83\% ( \pm 30,93), mas também baixa média anual de consultas médicas $(2,05 \pm 1,19)$ comparado com outros estados como São Paulo [16].

As desigualdades sociais no Brasil têm relação significativa com as condiçóes de saúde, sendo que em locais de pior nível socioeconômico se encontram piores condiçôes de saúde [17].

Os indicadores socioeconômicos, demográficos e de saúde utilizados no agrupamento dos municípios mineiros foram escolhidos por contemplarem esses aspectos de maneira combinada. A análise de segmentação pressupóe a formação de um conjunto de objetos no qual cada elemento está mais próximo e é mais similar a elementos dentro do agrupamento do que qualquer outro fora do grupo [11].

Outras variáveis contextuais como os aspectos demográficos, aspectos relacionados à infraestrutura física e humana dos serviços de saúde, o investimento público em saúde, não estáo disponíveis para todos os municípios do estado e, por isso, não puderam ser avaliadas. Além disso, os indicadores selecionados correspondem aos resultados do censo de 2000, uma vez que houve apenas divulgação parcial e agregada dos dados do censo de 2010.

Por isso, a interpretação dos achados do presente estudo deve ser feita com cautela, mas serve para ser usada como exploração de possíveis relaçóes entre os indicadores em questão. Ainda, assim, a análise de segmentação foi uma ferramenta muito útil para esse tipo de amostra, pois pequenas particularidades de cada município apareceram durante o processo de agrupamento em diferentes subconjuntos. Essa análise estatística é capaz de detectar agrupamentos homogêneos em um conjunto de dados [11].

$\mathrm{O}$ agrupamento 1 reuniu municípios com piores indicadores socioeconômicos, maior densidade demográfica e maior índice de Gini. Já o segundo agrupamento reuniu aqueles como de melhores indicadores.

Em relação aos indicadores de saúde, pôde-se observar uma tendência favorável ao princípio de equidade, pois os municípios com piores indicadores socioeconômicos apresentaram maior cobertura da ESF. Ainda que estes resultados sejam exploratórios, percebe-se que a Saúde da Família pode estar contribuindo para a reduçáo da desigualdade em relação aos indicadores socioeconômicos.

Apesar de o emprego dos estudos com análise de segmentação ser incipiente na área da saúde, esse resultado é semelhante ao observado no país, sendo que quanto mais baixo os indicadores sociais maior o nível de cobertura pela ESF [18].

Por outro lado, não houve diferenças percentuais significantes entre os agrupamentos em relação ao IDH e índice de Theil, o que pode indicar que con- 
dições de desenvolvimento municipais e desigualdade não corresponderam às conhecidas diferenças entre os municípios de um mesmo estado, quando as informaçóes são analisadas individualmente [19].

O Brasil é um país com inúmeras realidades sociais e demográficas, além de processos diferenciais de organização da assistência à saúde. Portanto, é plausível ter-se diferenças na implantação da Estratégia de Saúde da Família, evidenciadas nas diferenças entre seus indicadores de saúde [20]. Os serviços de atenção básica cumprem duas funções complementares. De um lado, há evidências de que cuidados básicos podem evitar o agravamento de problemas de saúde e reduzir a demanda a serviços de maior complexidade. De outro, geram demanda para serviços hospitalares [21].

Neste estudo investigou-se o efeito dos fatores demográficos e socioeconômicos no padrão de mortalidade hospitalar dos municípios de Minas Gerais relacionando-os ao sexo e à idade. Apesar de não ser possível realizar inferência estatística sobre os dados, observou-se que existem diferenças entre os indicadores municipais e a mortalidade para ambos os sexos.

Os padróes de mortalidade variam de acordo com os diferenciais sociais e econômicos [22]. Entretanto, comportamentos específicos do homem e da mulher, que explicam as diferenças entre o acometimento por doenças, e ainda causas externas relacionadas principalmente a violência e acidentes de trânsito [23], não foram avaliadas neste estudo.

Os resultados indicam também que a mortalidade hospitalar para o sexo masculino é maior que para o sexo feminino, independentemente do agrupamento em que os municípios sáo classificados. Isto significa dizer que as diferenças de mortalidade entre os sexos parecem náo ser explicadas apenas pelos indicadores em questão. Nos países desenvolvidos se percebe um processo de redução do diferencial de mortalidade por sexo [24].

Os problemas relacionados à utilização de dados secundários são bem definidos na literatura científica, tais como fragilidades nos registros relativos ao controle de qualidade dessas informaçóes nas esferas estadual e federal [25-27]. Esses problemas poderiam ser minimizados pelo correto preenchimento das declaraçôes de óbitos e transmissão segura dos dados.

A proporção de óbitos de origem mal definidas entre a população de 60 anos ou mais ainda é elevada, sendo que a qualidade das informaçóes é baixa e afeta sobremaneira a análise da mortalidade de causas específicas [3]. Além disso, apesar de a mortalidade hospitalar ser um indicador de desempenho tradicional, calculado por meio de bases de dados administrativas, pode haver subestimação dos dados, uma vez que não são computados para o cálculo desse indicador os pacientes que vão a óbito após a alta hospitalar [28-29].

\section{Conclusão}

Pode-se concluir que não há diferenças entre os dois grupos de municípios quando comparados à taxa de mortalidade de idosos por sexo. Mesmo assim, este estudo é importante, pois é um dos poucos a utilizar a análise de segmentaçáo com dados secundários. Percebeu-se que os indicadores IDH e índice de Theil não explicam as diferenças socioeconômicas e demográficas entre os municípios de Minas Gerais. Essas informaçôes poderão fornecer indícios para a criação de estratégias nos programas de saúde do idoso. Outros estudos poderão ser realizados na investigação da possível relação entre condições socioeconômicas dos municípios e as diferenças existentes entre a mortalidade hospitalar entre os sexos.

\section{Agradecimentos}

Os autores agradecem a Oldac Viana Campos pela participaçáo na coleta e construçáo do banco de dados de dados.

\section{Referências}

1. Camarano AA, Beltrão KI, Araújo HE, Pinto MS. Transformaçóes no padrão etário da mortalidade brasileira em 1979-1994 e no impacto na força de trabalho. Brasília: Ipea; 1997.

2. IBGE. Instituto Brasileiro de Geografia. Coordenação de População e Indicadores Sociais: Breves notas sobre a mortalidade no Brasil no período de 2000-2005. Rio de Janeiro: Diretoria de Pesquisa; 2006.

3. Camarano AM, Kanso S, Mello JL. Como vai a população brasileira? A mortalidade da população idosa no Brasil. In: BRASIL. Instituto de Pesquisa Econômica Aplicada. Os Novos Idosos Brasileiros: Muito Além dos 60? Brasília: Ipea; 2004. p. 25-73.

4. Silva MC. O processo de envelhecimento no Brasil: desafios e perspectivas. Textos Envelhecimento 2005;8(1):43:60.

5. Mendes MRSSB, Gusmão JL, Faro ACM, Leite RCBO. A situação social do idoso no Brasil: uma breve consideração. Acta Paul Enferm 2005;18(4):422-6. 
6. Drumond Junior M, Barros MBA. Desigualdades socioespaciais na mortalidade do adulto no município de São Paulo. Rev Bras Epidemiol 1999;2(1/2):34-49.

7. Organizaçâo Pan-Americana da Saúde. Indicadores básicos para a saúde no Brasil: conceitos e aplicaçóes. Rede Interagencial de Informação para a Saúde [Internet]. 2a ed. Brasília: Organização Pan-Americana da Saúde; 2006.

8. Brasil. Fundação João Pinheiro e Instituto de Pesquisas Econômicas Aplicadas. Definiçáo e metodologia de cálculo dos Indicadores e Índices de Desenvolvimento Humano e condiçóes de vida. Atlas de Desenvolvimento Humano no Brasil, 1998. [citado 2011 Jul 28]. Disponível em: URL: http://www.HDR2000/ Metodologias.

9. Ramos L. Interpretando variaçôes nos índices de desigualdade de Theil. Pesq Plan Econ 1990;20(3):479-88.

10. Minas Gerais. Datagerais. Estudos Sociais. [citado 2012 Jun 28]. Disponível em: URL: http://www.datagerais. mg.gov.br/datagerais/site/index.php.

11. Hair JF, Black WC, Babin JB, Anderson RE, Tatham RL. Análise multivariada de dados. $6^{\mathrm{a}}$ ed. Porto Alegre: Artmed; 2009.

12. Camarano AA, Beltrão KI, Neupert R. Século XXI: a quantas andará a população brasileira. In: Ipea/Iplan. Para a década de 90: prioridades e perspectivas de políticas públicas populaçáo, emprego, desenvolvimento urbano e regional. Brasília: Ipea/Iplan; 1990. p. 1-36.

13. PNUD. Programa das Naçôes Unidas para o Desenvolvimento. IDH 2011 cobre número recorde de 187 países e territórios. [citado 2012 Jun 27]. Disponível em: URL: http://www.pnud.org.br/Noticia.aspx?id=2589

14. Nasser B. Economia Regional, Desigualdade Regional no Brasil e o Estudo dos Eixos Nacionais de Integração e Desenvolvimento. Revista do BNDES [Internet]. 2000;7(14). [citado 2012 Jun 27]. Disponível em: URL: http://www.bndes.gov.br.

15. Barros RP, Mendonça RS. Os determinantes da desigualdade no Brasil. In: IPEA. Economia brasileira em perspectiva. Rio de Janeiro: Ipea; 1996. p.421-74.

16. Rehem TCMSB, Egry EY. Internaçóes por condiçóes sensíveis à atençáo primária no Estado de São Paulo. Texto Contexto Enferm 2012;21(3):4755-66.

17. Szwarcwald CL, Bastos FI, Barcellos C, Pina MF, Esteves MAP. Health conditions and residential concentration of poverty: a study in Rio de Janeiro, Brazil. J Epidemiol Community Health 2000;54(7):530-6.
18. Ministério da Saúde. Secretaria de Atenção à Saúde. Departamento de Atenção Básica. Saúde da família no Brasil: uma análise de indicadores selecionados: 1998-2005/2006. Brasília: Ministério da Saúde; 2008.

19. Aquino R, Barreto ML. Programa Saúde da Família: acerca da adequação do uso do seu indicador de cobertura. Cad Saúde Pública 2008;24(4):905-14.

20. Campos FE, Aguiar RAT, Oliveira VB. O desafio da expansão do programa de Saúde da Família nas grandes capitais Brasileiras. Physis: Rev Saúde Coletiva 2002;12(1):47-58.

21. Oliveira EXG, Travassos C, Carvalho MS. Acesso à internaçáo hospitalar nos municípios brasileiros em 2000: territórios do Sistema Único de Saúde. Cad Saúde Pública 2004;20(Supl 2):298-309.

22. Laurenti R, Jorge MHPM, Gotlieb SLD. Perfil epidemiológico da morbi-mortalidade masculina. Ciênc Saúde Coletiva 2005;10(1):35-46.

23. Paes-Sousa R. Diferenciais intra-urbanos de mortalidade em Belo Horizonte, Minas Gerais, Brasil, 1994: revisitando o debate sobre transiçóes demográfica e epidemiológica. Cad Saúde Pública 2002;18(5):110-14.

24. Siviero PC, Turra CM, Rodrigues RN. Diferenciais de mortalidade por sexo: notas para estudo. (Texto para discussão; 408). Belo Horizonte: UFMG/Cedeplar; 2010.

25. Paiva NS, Coeli CM, Moreno AB, Guimarães RM, Camargo Júnior KR. Sistema de informaçóes sobre nascidos vivos: um estudo de revisão. Ciênc Saúde Coletiva 2011;16(Supl. 1):1211-20.

26. Drumond EF, Machado CJ, Vasconcelos MR, França E. Utilização de dados secundários do SIM, Sinasc e SIH na produção científica brasileira de 1990 a 2006. Rev Bras Estud Popul 2009;26(1):7-19.

27. Mello JMHP, Gotlieb SLD. O sistema de informaçáo de atenção básica como fonte de dados para os sistemas de informaçóes sobre mortalidade e sobre nascidos vivos. Inf Epidemiol SUS 2001;10(1):7-18.

28. Pinheiro RS, Vieira RA, Coeli CM, Vidal EIO, Camargo Jr KR. Utilizaçáo do SIH-SUS e do SIM para o cálculo da mortalidade hospitalar e em 30 dias para as internaçóes de pacientes com fratura proximal de fêmur. Cad Saúde Coletiva 2006;14(2):337-344.

29. Travassos C, Noronha JC, Martins M. Mortalidade hospitalar como indicador de qualidade: uma revisão. Ciênc Saúde Coletiva 1999;4(2):367-81. 